TENDENCIAS

Revista de la Facultad de Ciencias Económicas y Administrativas. Universidad de Nariño

Vol. XV. No. 1 - 1er. Semestre 2014

Enero-Junio - Páginas 228-241

\title{
CONCEPTOS Y PRINCIPIOS DE ECONOMÍA Y METODOLOGÍAS UTILIZADAS EN LA INVESTIGACIÓN ECONÓMICA
}

La Economía es el arte de sacar el mayor provecho de la vida.

George Bernard Shaw

Por: Roberto Posso Ordóñez ${ }^{1}$

\section{RESUMEN}

Este ensayo se cataloga como descriptivo, debido a que desarrolla temas vinculados con los fenómenos de la ciencia económica. Está estructurado en dos partes: en la primera encontraremos una síntesis de las principales definiciones de Ciencia Económica cuyos autores pertenecen a diferentes escuelas de pensamiento (clásica, mercantilista, neoclásica, monetarista y algunos economistas contemporáneos). La segunda parte contiene un breve análisis de los aportes hechos por algunos de los grandes matemáticos, cuyos conocimientos alimentan las nuevas teorías que desarrollan los investigadores contemporáneos, así como las diferentes metodologías utilizadas por los distintos economistas que en el pasado elaboraron, desarrollaron y crearon conceptos, leyes y principios que condujeron a determinar los objetivos de la ciencia económica.

Palabras clave: Rol de la economía, Metodología utilizada por Economistas, Modelos matemáticos.

Clasificación JEL: A11, B41, C20.

1. Catedrático de la Universidad de los Hemisferios, Quito, Ecuador - Consultor Económico. Correo electrónico: rposso.ec@gmail.com

Fecha de recepción: 15 de noviembre de 2013 - Fecha de aprobación definitiva: 21 de febrero de 2014 


\title{
ECONOMIC CONCEPTS AND PRINCIPLES AND METHODOLOGIES USED IN ECONOMIC RESEARCH
}

\author{
By: Roberto Posso-Ordóñez
}

\section{ABSTRACT}

This essay could be classified as descriptive, since it deals with topics concerning the economic field. It is structured in two parts: the first one presents a summary of the different definitions of Economy as a science, definitions provided by economists belonging to the different schools and trends of thought starting with (classics, commercialists, neo-classics, monetarism and finishing up with some of the contemporary ones). The second part is a brief analysis of the contributions made by some of the great mathematicians, which support the new theories developed by contemporary researchers as well as the different methodologies used by the different economists that created, developed and structured laws and principles that allowed the objectives of Economy as a science to be determined.

Key words: Role of the economics, Economic Mythology Mathematical Models.

JEL Classification: A11, B41, C20. 


\section{INTRODUCCIÓN}

En marzo de 2001 se dio el estallido de la burbuja financiera de la llamada "economía.punto.com" y de inmediato el capital se desplazó al campo de los préstamos hipotecarios y en junio de 2008 se dio el colapso de la "burbuja inmobiliaria" que fue provocada por el no pago de los préstamos hipotecarios conocidos como "subprime". Para muchos economistas la crisis del 2008 es considerada como la peor que haya afectado a la humanidad, incluida la ocurrida en la década de los años 30 del siglo pasado. El no pago de los mencionados préstamos hipotecarios provocó, entre otros efectos, la bancarrota del banco de inversiones Lehman Brothers y, por un efecto dominó, contagió a las bolsas de valores, ocasionando una crisis bursátil que si bien se inició en los Estados Unidos, después se propagó por todo el mundo. Igualmente se registró una reducción de los volúmenes de crédito, se incrementaron las tasas de desempleo y, en conjunto, produjo una crisis económica a escala mundial. El impacto también se extendió al sector agrícola ocasionando una crisis alimentaria global (bbc.co.uk: 2009).

A lo señalado anteriormente hay que añadir los cambios políticos y sociales que se están implementando, desde comienzos de este siglo XXI, en los países de América Latina. Estas reformas y los hechos ocurridos en torno a la "Primavera Árabe”, que vivieron algunos países productores de petróleo, inciden directamente en las actividades productivas y financieras de los países de la Región.

Vivimos en un mundo globalizado, "en donde opera la muda razón del capital, que no tiene patria, sino intereses". (...) "Ya no se trata únicamente de la renta tradicional de la tierra, ampliada al control de los recursos naturales, sino de la nueva forma de la renta, bajo la forma de renta del conocimiento, renta tecnológica”. (Villavicencio, 2013:32).

Dada esta nueva coyuntura internacional nació la inquietud de traer a la memoria dos aspectos que parecen importantes recordar para entender la problemática económica actual. Para el efecto se estima conveniente mencionar, por una parte, las principales categorías de conocimiento que se incorporan en las diferentes concepciones de la Economía y, por otra, sugerir para el proceso de investigación en Economía, el uso del método cuantitativo -sin que éste sea excluyente de otras posibilidades metodológicas- como el camino idóneo para interpretar y pronosticar la realidad y luego, eventualmente, construir un conocimiento teórico que explique el porqué de las cosas.

Como consecuencia de lo dicho anteriormente, este ensayo podría catalogarse como descriptivo, debido a que desarrolla temas vinculados con los fenómenos de la ciencia económica. Está estructurado en dos partes: en la primera encontraremos una síntesis de las principales definiciones de Ciencia Económica que dieran economistas pertenecientes a diferentes escuelas de pensamiento que surgieron alrededor de esta temática, estos son los clásicos, los mercantilistas, los neoclásicos, los monetaristas y algunos contemporáneos. La segunda parte contiene un breve análisis de los aportes hechos por algunos de los grandes matemáticos, cuyos conocimientos alimentan las nuevas teorías que desarrollan los investigadores contemporáneos, así como las diferentes metodologías utilizadas por los distintos 
economistas que en el pasado elaboraron, desarrollaron y crearon conceptos, leyes y principios que condujeron a determinar los objetivos de la ciencia económica.

En este trabajo se utilizan fuentes de información secundaria, es decir documental.

\section{ALGUNOS CONCEPTOS Y PRINCIPIOS DE ECONOMÍA}

Resulta bastante complicado el encontrar una definición única de la ciencia económica y en consecuencia su objeto de estudio puede aparecer impreciso. Lo afirmado obedece a que tanto los padres de la economía como los economistas de las recientes escuelas de pensamiento no se han puesto de acuerdo sobre la temática debido a los diferentes enfoques teóricos que caracterizan a cada una de las escuelas de pensamiento. Por ejemplo, Gary Becker² en su obra "El enfoque económico de la conducta humana" plantea que "La definición de Economía en términos de bienes materiales es la más limitada y menos satisfactoria. No descubre adecuadamente el mercado ni lo que los economistas 'hacen' (...). La definición de economía en términos de medios escasos y usos alternativos del mercado es la más general de todas. Define la economía basándose en la naturaleza del problema que se trata de resolver, y abarca mucho más que el sector del mercado o 'lo que hacen los economistas' (...) Esta definición de economía es tan amplia que a menudo es una fuente de descontento más que de orgullo para muchos economistas y frecuentemente se considera de forma inmediata que excluye la mayor parte del comportamiento ajeno al mercado. Todas estas definiciones de economía simplemente defienden su ámbito, pero ninguna nos da la menor información acerca de lo que es el enfoque 'económico'. (...) Separémonos, por tanto, de las definiciones, porque creo que lo mejor que distingue a la economía como disciplina de otras disciplinas en las ciencias sociales no es su objetivo, sino su enfoque" (Becker, 1998: 507).

Veamos cómo piensan sobre la Economía algunos de los economistas más renombrados:

Tradicionalmente se acepta que la economía apareció como ciencia con la publicación de la obra de Adam Smith (1723-1790) intitulada "La riqueza de las Naciones” (1776). Este filósofo es considerado como el padre de la ciencia económica y sus ideas son el fundamento de la economía moderna debido a que "defiende el principio de división del trabajo y libertad de comercio. Smith pensaba que la satisfacción del propio interés individual, limitado por el de los demás, es el mejor medio para conseguir el mayor beneficio para el mayor número de gente. Sin embargo, Smith apoyó la intervención del Estado en materia de justicia, educación, salud y todas aquellas empresas que la iniciativa privada fuese incapaz de abordar" (Garban: 5). Según Aguirre (1962:41), Smith realiza su investigación basándose en la teoría del valor trabajo y muchas veces por las simples apariencias.

David Ricardo (1772-1823), en su libro "Principios de economía política y tributación” (1817), afirma que el principal problema de la economía política es determinar las leyes que rigen la distribución del ingreso entre los diferentes

2. Premio Nobel de Economía 1992. 
agentes económicos. "Sin embargo, eran los cambios en la distribución funcional del ingreso a través del tiempo los que concentraban su atención, así que empezó planteando una teoría que pudiera explicar las ganancias, los intereses, las rentas y los salarios" (Guerrero, 2001).

Con este propósito desarrolló la teoría del valor trabajo. Se preocupó también de averiguar las causas del crecimiento o, si se prefiere, del origen de la riqueza de los países a través del análisis de los factores que explican la distribución de la renta. Así, este autor, dentro de sus múltiples contribuciones a la economía, lo hizo en el campo de la teoría del comercio internacional, al haber desarrollado el principio de la ventaja comparativa, a través de la cual explica la especialización que buscan los países para mejorar sus relaciones comerciales. En sus investigaciones utilizó los métodos deductivo y abstracto (Aguirre, 1962: 155).

El aporte más importante de John Stuart Mill (1806-1873), autor del libro "Principios de Economía política con algunas de sus aplicaciones a la filosofía social" (1848), consiste en su afirmación de que “...Las proposiciones de la economía, sólo son ciertas en la medida en que sea cierta la premisa de donde se han deducido, esto es, el postulado de que el hombre trata de obtener el máximo de riqueza con el menor trabajo posible. Por ello, decía, en la aplicación práctica de las proposiciones económicas es necesario siempre tener en cuenta si el postulado fundamental de la economía se verifica en la realidad y en qué medida lo hace; en otros términos, si la conducta humana está guiada por principios distintos del adoptado por la ciencia económica como premisa a sus deducciones y la medida en que lo hace" (Napoleoni, 1962: 649).

Por otra parte, el mismo autor sostiene que el objeto de la economía está en la diferenciación que existe entre las leyes de la producción y de la distribución. Al respecto manifiesta que las primeras son inmutables debido a su carácter natural, las mismas no pueden ser cambiadas por los humanos. Sobre las leyes de la distribución Mill afirma que son producto de arreglos sociales y en sí, son las instituciones las que las construyen y realizan la distribución. En este punto se diferenciaba de la gran mayoría de pensadores clásicos quienes construyen un sistema que fue utilizado en la política para cerrar los caminos a las masas oprimidas ya que según esta no había forma de mejorar la retribución al trabajador pese a la buena voluntad que se tuviera (Guerrero, 2001).

William Stanley Jevons (1835-1882) introdujo cambios importantes en el concepto de Economía. Es uno de los pioneros de la escuela marginalista ${ }^{3}$ y su contribución más importante quizá radica en el hecho de que él postula el empleo de modelos matemáticos y estadísticos en el desarrollo científico de la economía. Igualmente sus análisis dan énfasis al enfoque microeconómico antes que el macroeconómico y consolida de modo explícito los principios hedonistas como fin supremo de la vida. En consecuencia, la economía puede definirse como "la ciencia que estudia las condiciones que debe satisfacer la conducta humana para conseguir un placer

3. La teoría marginalità o de marginalidad social, desechó la del valor-trabajo que heredó de la escuela clásica. Se enfoca — por medio de la subjetividad — en la satisfacción del consumidor, concepto al que denominó utilidad marginal. 
máximo con un costo mínimo” (Napoleoni, 1962: 651). Jevons optó por las pruebas empíricas en sus investigaciones.

Alfred Marshall (1842-1924), autor de "Principios de Economía" es considerado, junto a Léon Walras, como los padres de la economía ortodoxa moderna. Para Marshall la economía política o economía, es el estudio de la humanidad en las ocupaciones ordinarias de la vida; examina esa parte de la acción individual y social que está más estrechamente conectada con la obtención y el uso de los requisitos materiales del bienestar, por lo que se le considera el precursor de la Economía del Bienestar ${ }^{4}$. Marshall fue uno de los primeros autores en introducir la variable tiempo para hacer análisis en la economía. Consciente de la multitud de interrelaciones que existen en la actividad económica, trató de diseñar un modelo analítico, el "Equilibrio parcial", cuya finalidad era aislar el comportamiento de un determinado aspecto económico, suponiendo que todo lo restante permanece invariable. Es así como surgió el concepto Ceteris Paribus, término ampliamente utilizado en la economía actual para reflejar en un análisis que "todo el resto permanece constante" (Marshall, 1931).

Según la Universitat de las Illes Baleares, la obra de Arthur Pigou (1877-1959) "La Economía del Bienestar” (1912) “...constituye una guía de toma de decisiones de política económica, toda vez que reconoce, que un político jamás debería ceñirse al mero razonamiento económico, sino que debería considerar todos los múltiples y ricos aspectos de la existencia humana”.

Su preocupación objetiva era la renta nacional y los problemas del desempleo. (Pensamiento económico de Cecil Pigou). Pensaba que el Estado podía hacer mucho para mejorar las condiciones de vida de sus habitantes, bajo el principio de que los mercados tienen imperfecciones que no les permiten funcionar eficientemente, pero también en la idea -bastante elitista, por cierto- de que el ciudadano medio no siempre está capacitado para tomar las decisiones que más le convienen (Universidad de Zaragoza).

Lionel Robbins (1898-1984), en su "Ensayo sobre la naturaleza y significación de la ciencia económica” (1932), manifiesta “...economía es una ciencia que estudia el comportamiento humano como relación entre los fines dados y los medios escasos que tienen aplicaciones alternativas” (Ludwig von Mises Institute: 2007). Esta afirmación tuvo gran acogida en los economistas e influyó notablemente en el planteamiento de la naturaleza y ámbito de la Economía.

En la actualidad la definición de Robbins, al referirse al comportamiento humano como una relación entre fines y medios escasos, susceptibles de usos alternativos, ha sido acogida con éxito por la comunidad de economistas debido a que proporciona un punto de partida a los estudios llevados a cabo mediante la aplicación de la lógica económica a los fenómenos sociales (Nolan, 2008).

4. La Universidad Berkeley acota: "Economía del bienestar: aproximación metodológica para juzgar la locación de recursos y establecer criterios para la intervención estatal”. 
Parece oportuno presentar ahora, la definición que utilizan Paul Samuelson (1915-2009) y William Nordhaus (1941-....), profesores de MIT ${ }^{5}$ y de Yale University, respectivamente, en sus casi veinte manuales introductorios de Economía escritos para cientos de generaciones de jóvenes aspirantes a graduarse de economistas y que textualmente reza: "La economía es el estudio de la manera en que las sociedades utilizan los recursos escasos para producir mercancías valiosas y distribuirlas entre los diferentes individuos" (Samuelson, 2010: 4). Como en esta definición subyacen dos conceptos importantes que son: la escasez y el concepto de eficiencia, ellos agregan: "La esencia de la teoría económica es reconocer la realidad de la escasez y luego encontrar la manera de organizar a la sociedad de tal forma que produzca el uso más eficiente de los recursos. Es ahí donde hace su contribución única” (Samuelson, 2010: 4 y 5).

Milton Friedman (1912-2006), es el principal representante de la llamada Escuela de Chicago, grupo de economistas que considera que los mercados competitivos y libres de la intervención del Estado, contribuyen a que el funcionamiento de la economía sea más eficiente. Sus postulados fueron la base de las políticas neoliberales que se establecieron en algunos países, en la década de 1980. Este economista define "La economía como positiva es un cuerpo de generalizaciones a título experimental acerca de los fenómenos económicos, que puede usarse para predecir las consecuencias de los cambios en las circunstancias que la rodea" (Ibero, et al: 2008).

\section{ALGUNAS CONSIDERACIONES SOBRE LA METODOLOGÍA APLICADA EN ECONOMÍA}

La interminable necesidad y curiosidad que tienen los seres humanos por descubrir cosas nuevas y de encontrar explicaciones lógicas de la realidad del mundo en el que está inmerso, les ha conducido a buscar el instrumento que cumpla esos vehementes deseos. Ese enlace entre el sujeto con el objeto de la investigación es el camino metodológico. Sin la metodología es casi imposible llegar a la lógica que conduzca al conocimiento científico.

Podemos definir la metodología como el conjunto de procedimientos lógicos a través de los cuales se plantean los problemas científicos y se ponen a prueba las hipótesis y los instrumentos de trabajo investigados.

Antes de analizar el pensamiento de algunos tratadistas que sugirieron la utilización de las matemáticas y estadística como método de investigación científica, recordemos esta frase de Galileo al expresar que: Las matemáticas son el alfabeto con el cual Dios ha escrito el universo.

Las matemáticas, en efecto, como procedimiento lógico han ayudado a comprender y profundizar los principios y leyes de la economía. Por ejemplo, el entender el efecto que producen los impuestos sobre el ingreso de capitales, y hasta la competencia para determinar qué puede depararnos el futuro, a través de la probabilidad. Recordemos que en la era postmoderna, las matemáticas están estrechamente vinculadas a nuestras vidas y a las acciones cotidianas -como el

5. Massachusetts Institute of Technology. 
comercio por internet, la comunicación virtual y hasta los vuelos espaciales- son producto de los avances del pensamiento matemático.

Sin el desarrollo de los conceptos de probabilidad y estadística llevados a cabo, entre otros científicos, por Galileo Galilei (1564-1642), que creó la teoría de la media de errores y puso las bases para el nacimiento de la estadística; por Joseph Lagrange (1749-1827) que evidenció que existen analogías entre los juegos de azar y los fenómenos estocásticos en física y ciencias sociales; por Karl Gauss (1777-1855), creador de la distribución gaussiana, y muchos otros intelectuales, las ciencias tendrían menos peso o podrían no haber tenido la importancia que tienen ahora (Chipia, et al, 2).

Las leyes del pensamiento de George Boole (1815-1864) "demostraron que la lógica era un sistema de procesos sujetos a leyes idénticas a las del álgebra, lo que permite inferir que la computación digital es de extraordinaria ayuda para probar hipótesis de los modelos utilizados en economía” (Hawking, 2007: XIV).

Por su parte, Kurt Gödel (1906-1978) hizo un aporte muy importante, a través de su demostración de un teorema de "Cálculo de predicados" que hizo recapacitar a la comunidad científica respecto a la relatividad que caracteriza a la verdad. Su tesis doctoral conduce a un doble resultado que se sintetiza en las sabias palabras que se transcriben a continuación: "en un sistema lógico suficientemente complejo (como el de la aritmética) existen enunciados que no pueden ser probados ni refutados. Por si fuera poco, también probó que la cuestión de si un sistema en si mismo era lógicamente consistente no podía tampoco ser demostrado dentro de los límites del propio sistema” (Hawking, 2007: XIV).

Otro aspecto importante que hay que resaltar es el hecho de que Gödel distinguió claramente que "demostrabilidad" y "verdad" no son lo mismo. Este concepto permite llegar a determinar hasta qué punto las investigaciones que se realizan son prácticas o no, es decir si pueden ser o no aplicadas en la vida real.

"En definitiva, descubrió que existían afirmaciones verdaderas que no podían ser probadas dentro del sistema" (Entrena).

En tratándose de la metodología en la Economía se puede apreciar que las corrientes dominantes para la filosofía tuvieron una gran influencia sobre los economistas a lo largo del tiempo. Los economistas clásicos (fines del siglo XVIII y principios del XIX) utilizaron el método inductivo. Recordemos que en la época de aquellos economistas, la inducción estaba teniendo éxito en las investigaciones realizadas en el campo de las ciencias naturales. Si bien Adam Smith está sujeto a diversas influencias metodológicas que le hacen utilizar una compleja mezcla de procedimientos, en último término prefiere utilizar claramente el método inductivo (Gómez: 2004).

David Ricardo, por el contrario, utilizó el método deductivo y la modelización abstracta en sus trabajos de investigación. En 1823, luego de su muerte, se da por primera vez un debate respecto del método utilizado por este economista y en ese debate se destaca Nassau Senior (1790-1864), quien establece una distinción entre los conceptos de economía como ciencia y como arte. La primera conceptualización 
sería la que corresponde a la actual definición de economía positiva ${ }^{6}$ mientras que la segunda coincide con la idea de economía normativa ${ }^{7}$.

Senior también señala que los principios generales de la economía han sido elaborados como resultado de la observación de la realidad, de la cual se han obtenido esas conclusiones. De esta forma, parece que el método deductivo va tomando asidero entre los economistas.

Por su parte, John Stuart Mill, concuerda con la idea de Senior en cuanto se refiere al método que debe usarse en economía. Estima que el método de la inducción debe aplicarse para las ciencias no sociales, concluyendo que la economía debe emplear el método deductivo debido a que el empleo del primero podría llevar a cometer errores de concepto por cuanto existen varias causas que afectan a un solo fenómeno económico. Considera que el economista debe comenzar la investigación partiendo de premisas sicológicas, a las que se llega por introspección. Luego, con esas premisas es necesario elaborar una teoría, la cual se somete a comprobación mediante procedimientos empíricos. Estos procedimientos posibilitan revelar las relaciones esenciales y las características fundamentales del objeto de estudio (IV. 3 John Stuart Mill).

John Eliot Cairnes (1823-1875) fue un ferviente seguidor de Mill. Su principal aporte pone énfasis en que la economía política es una ciencia hipotético-deductiva. El economista irlandés apoya las ideas de que la economía debe estructurarse sobre premisas reales, obtenidas de hechos evidentes que se obtienen a través de la introspección y no por la inducción. Cairnes adopta la posición de que la verificación de los hechos económicos solo se puede llevar a cabo de modo imperfecto, pero defiende que verificar ayuda a fortalecer el razonamiento deductivo. Aclara que la verificación no se la aplica para conocer si las hipótesis son verdaderas o falsas sino más bien como un método que permite establecer la factibilidad de aplicación o no de las teorías. Si una determinada teoría se ha deducido correctamente, será cierta. Si existen discrepancias entre la teoría y los hechos, puede interpretarse que existen causas perturbadoras que afectan a la teoría, debido a la aplicación de un método incorrecto. Pero la teoría será acertada si se ha obtenido con rigor científico, mediante la aplicación del proceso deductivo (Cairnes y Rodan, 2007: 49).

La coincidencia en la forma de pensar tanto de Senior y Mill como la de Cairnes, permite inferir que este hecho puede corresponder a la influencia que recibieron de la corriente de pensamiento utilitarista que estaba en boga en la Inglaterra del siglo XIX.

En la historia de la metodología económica, se registra que en la obra publicada por Lionel Robbins, el autor aboga por el uso del método deductivo en economía. Añade que el procedimiento inductivo no es acertado para la elaboración de teoría económica. El referido economista destaca tres aspectos que deben considerase al momento de decidir por el método a seguir:

6. La economía positiva estudia "qué es”. Busca explicar como los hechos se unen, busca formular y comprobar teorías que expliquen relaciones entre los factores económicos.

7. Fundamenta su análisis en un juicio de valor, entregando resultados respecto del "como debiera ser". 
a. La subjetividad de los individuos tiene papel importante, debido a que la conducta humana es intencional y no una mera repetición de procesos deterministas. La economía, por tanto, no puede desligarse de la sicología para elaborar teorías

b. La complejidad de la realidad impide que las condiciones iniciales permanezcan invariables en distintas situaciones.

c. La falta de uniformidad en la ocurrencia de los eventos resta eficacia a los procedimientos puramente empíricos, aunque éstos pueden ser útiles como punto de partida (Robbins, 1971: 149).

A mediados de la década de los años 1930 se aprecian los primeros síntomas que denotan la influencia del falsacionismo en el campo de la metodología económica. Su principal representante fue Karl Popper (1902-1994) conocido por sus aportaciones epistemológicas sobre metodología de la ciencia económica. Este prestigioso economista austriaco publicó en 1935 el libro "La lógica de la investigación científica" en el cual el método inductivo y en general cualquier método que sea partidario de aplicar el criterio de verificación empírica de las teorías, Roberto Gómez, expresa textualmente:

La principal aportación metodológica de Popper, puede resumirse de la siguiente forma, aunque una teoría no puede ser verificada, sí puede ser falsada, es decir, si el conjunto de observaciones favorables no puede demostrar la veracidad de una teoría, un hecho contrario a ella, puede demostrar que la teoría es falsa. A partir de aquí, Popper establece un criterio de demarcación, distinguiendo entre la ciencia y la no-ciencia, concluyendo que a una teoría se le otorga el carácter de científica si es susceptible de ser falsada, en caso contrario, no es científica: "Estas consideraciones nos sugieren que el criterio de demarcación que hemos de adoptar no es el de la verificabilidad, sino el de la falsabilidad de los sistemas. Dicho de otro modo, no exigiré que un sistema científico pueda ser seleccionado, de una vez para siempre, en un sentido positivo, pero sí que sea susceptible de selección en un sentido negativo por medio de contrastes o pruebas empíricas, ha de ser posible refutar por la experiencia un sistema científico empírico" (eumed.net).

Una crítica desde una posición de positivismo lógico argumentaba que la mayoría de las teorías económicas no eran más que tautologías ${ }^{8}$ basadas en el supuesto del conocimiento perfecto, incluyendo el conocimiento perfecto del futuro. Proponía en cambio que la ciencia económica debería consistir en proposiciones comprobables, 'falseables' en el sentido popperiano (Martínez, 2001).

Hutchinson, por su parte, hace una clara distinción entre lo que es y no es ciencia, señalando que ciencia debe ser concebiblemente capaz de ser sometida a comprobaciones empíricas "o ser susceptibles de ser reducidas -por deducción, lógica o matemática- a proposiciones contrastables” (Hutchinson, 1941).

8. Repetición inútil y viciosa de un mismo pensamiento, expresado de distintas maneras. 
Por otra parte Samuelson y Nordhaus también hicieron importantes aportes a la metodología de la economía, muy influenciados y vinculados con al escuela filosófica del operacionalismo ${ }^{9}$ (Jáuregui: 2001). Los autores destacan que el centro de la actividad de los economistas debe ser:

a) La elaboración de "teoremas operacionalmente significativos". Esto significa que las hipótesis vinculadas con datos empíricos puedan ser refutadas, aunque solamente sea en condiciones ideales.

b) El análisis del comportamiento dinámico puede proporcionar conocimiento sobre la estética comparativa del modelo. Esto es lo que Samuelson llama el "principio de correspondencia" (García, 2004: 6).

c) Quizá lo más importante parte del núcleo de la actividad de los economistas es que la ciencia debe elaborar no una explicación de la realidad sino una descripción de la misma.

Pocos años más tarde aparecerá el aporte metodológico de la economía positiva, liderado por Milton Friedman. El autor es reiterativo en expresar que el método inductivo no garantiza que la afirmación general o conclusión que se obtiene a partir de la observación de casos particulares, sea necesariamente verdadera, debido a que no existe una lógica inductiva.

En resumen, el economista monetarista sostenía, de manera contundente, que:

a) El objetivo de la ciencia es controlar la naturaleza.

b) La forma de alcanzar ese objetivo es a través de la experiencia. Por tanto el punto de partida en la elaboración de hipótesis debe ser la evidencia empírica y el de llegada, la realidad, porque es necesario contrastar las contradicciones de la teoría. El autor pone énfasis en el hecho de que una teoría será correcta si predice adecuadamente.

c) La validez de la teoría se confirma por la utilidad que preste y no por el hecho de que sea verdadera o falsa. El criterio definitivo para juzgar la validez de una teoría es la conformidad de sus predicciones.

d) El realismo de los supuestos no es necesario para que la teoría prediga correctamente.

e) La evidencia empírica puede refutar una hipótesis pero no probarla, de manera que la ciencia siempre tiene un carácter provisional (Ibero, et al: 2008).

\section{ALGUNAS REFLEXIONES}

El objetivo de la economía es el de formular interrogantes adecuadas sobre la realidad. De esta manera, las respuestas adecuadas permitirán tener un mejor conocimiento de la realidad objeto de estudio.

9. Propugnado por Bridgman, intenta solucionar el problema de la definición de los conceptos usados por las ciencias realizando una serie de "operaciones" prácticas o mentales 
Los métodos más utilizados para el análisis económico parecen ser tanto el inductivo como el deductivo. En muchos casos las teorías han sido elaboradas partiendo de la observación de la realidad. En otras ocasiones el empleo del método deductivo ha permitido encontrar respuestas a los interrogantes planteados.

El contraste empírico de la teoría, en términos popperianos, permite falsarla o aceptarla de modo provisional, esto por cuanto la teoría es transitoria y puede ser sustituida por otra teoría alternativa y con mejor armonía con la misma evidencia empírica anterior o con una nueva evidencia.

La utilización de métodos cuantitativos para responder los cuestionamientos respecto de la realidad económica y para hacer predicciones, parecen ser de los más idóneos. Para llevar con rigor científico las investigaciones en economía los instrumentos indicados son las matemáticas, la estadística y la econometría.

La aplicación de métodos cuantitativos en la investigación tiene las ventajas que se indican a continuación: requiere una visión objetiva del problema, estudia conductas y otros fenómenos observables, genera datos numéricos para representar el ambiente social, emplea conceptos preconcebidos y teorías para determinar qué datos van a ser recolectados, emplea métodos estadísticos para analizar las variables, emplea procedimientos de inferencia estadística para generalizar las conclusiones de una muestra a una población definida, es confirmatoria, inferencial y deductiva.

Entonces, el método cuantitativo requiere que exista una relación entre variables, cuya naturaleza pueda ser representada por algún modelo numérico. En la actualidad es posible medir todas las variables, sea en forma cuantitativa o cualitativa. Incluso las variables cualitativas pueden convertirse en cuantitativas. Esto permite obtener resultados descriptivos que pueden ser generalizados y sobre todo permite "predecir" el eventual comportamiento de las variables analizadas.

Dentro de la investigación cuantitativa tenemos la posibilidad de utilizar las modalidades: descriptiva, analítica y experimental. En la primera de las nombradas, no se requiere plantear una hipótesis, mientras que en la segunda se debe plantear una o varias que deberán ser probadas o negadas y en la tercera, la experimental, se ha ideado con el propósito de determinar, con la mayor confiabilidad posible, las relaciones de causa-efecto, para lo cual uno o más grupos, -llamados experimentales- se exponen a los estímulos experimentales y los comportamientos resultantes se comparan con los comportamientos de ese u otros grupos, llamados de control, que no reciben el tratamiento o estímulo experimental.

Para terminar, recordemos lo que el estadounidense Richard Feynman, una de las grandes personalidades de la historia de la física del siglo XX, manifestó sobre las matemáticas: Las matemáticas son uno de los descubrimientos de la humanidad. Por tanto no pueden ser más complicadas de lo que los hombres son capaces de comprender. 


\section{REFERENCIAS}

AGUIRRE, Manuel Agustín (1962). Apuntes para el estudio de la Historia del Pensamiento Económico. Los Clásicos y Marx. Editorial Universitaria, Quito, Tomo II, 709 p.

AUTAURCO, Lizeth (2010). Ensayo sobre la naturaleza y significación de la ciencia económica, en http:// milian-lizeth.blogsopt.com/2010/01/ensayo-sobre-la-naturaleza-y.html

BECKER, Gary (1998). El enfoque económico de la conducta humana, Anuario de Filosofía Jurídica y Social, No. 16, Universidad Diego Portales, Valparaíso, pp. 507-527 (traducción de la fundación chilena Fernando Fueyo).

CAIRNES, John, RODAN, ROSEISTEN (2007). El método de carácter y la lógica de la economía política. Editor W. McGee 1869 digitalizado 20 julio 2007, 184 p. En: http://www.books.google.es/.../The_cracter_and_logical_meth...consulta 12 agosto 2013

CHIPIA, Joan, CALLES, Ana, SUÁREZ, Eudiver (2010). Historia de la Probabilidad y Estadística. Universidad de los Andes, Facultad de Humanidades y Educación, p. 39.

ENTRENA, Carlos (2013). Biografía de Kurt Godel-Sociedad Andaluza de Educación en thales.cica.es/rd97/ Biografías/ consulta 22 septiembre 2013.

GARBAN, Jack. La riqueza de las naciones de Adam Smith, libro III, Universidad Central de Venezuela.

GARCÍA-COBIAN, Ramón (2004). El principio de correspondencia de Samuelson, documento de trabajo 235, Pontificia Universidad Católica del Perú, p. 9. Consulta 9 octubre 2013.

GÓMEZ, Roberto (2004). Evolución Científica y Metodológica de la Economía. En www.eumed.net/ cursecon/libreria/pedir-rgl-audit.html, consulta 1 octubre 20013. En: www.eumedGrandesEconomistas. Grandes Economistas-Nassau William Senior (1790...).

GUERRERO, Manuel (2001). Ciclo de Pensamiento Económico en Gestiopolis.com, David Ricardo (17721828) en www.gestiopilis.com/canales/economia/artículos, Consulta 24 julio 2010.

GUERRERO, Manuel (2001). Ciclo de pensamiento Económico en Gestiopolis.com, Los clásicos: John Stuart Mill (1806-1873). En www.gestiopolis.com/canales/economia/artículos/no8/ Consulta 24 de julio 2010.

HAWKING, Stephen (2007). Dios creó los números, Edición comentada, cuarta reimpresión, Egedsa, España, 1440 p.

HUTCHINSON, Terence (1941). The significance and basic postulates of economic theory, Journal of Political Economy, Vol, 49, Chicago Journals.

IBERO, Francisco; BARREIRO, Moncho; MOLINA, Jaime. Panafreedom: la economía positiva según Milton Friedman http://blogspot/2008/12/la-economapositivasegn-milton.html consulta 14 agosto 2013.

IV.3 John Stuart Mill. Biblioteca digital Ilce en bibliotecadigital.ilce.edu.mx/sites/ciencia/volumen3/.../161/.../sec_29htm consulta 15 septiembre 2013.

JÁUREGUI, Inmaculada (2001). Cuestiones epistemológicas en Antropología. Universidad de San Antonio de Murcia, Gazeta de Antropología, Artículo 16 en www.ugr.es/pwlac Consulta 9 octubre 2013. 
LUDWIG VON MISES INSTITUTE, ADVANCING AUSTRIAN ECONOMICS, LIBERTY AND PEACE. Ensayo sobre la naturaleza y significación de la naturaleza de la ciencia económica en http://www.elcato.org/bibliotecadelalibertad/planificación-para-libertad-esencial-de-mises Consulta 30 septiembre 2013.

MARSHALL, Alfred (1931). Principios de Economía-Introducción al estudio de esta ciencia. Traducción de la octava edición inglesa, volumen primero, editorial El Consultor bibliográfico, España, 53 p.

MARTÍNEZ COLL, Juan Carlos, (2010). EUMEDNET Enciclopedia y Biblioteca Virtual, en http://www.eumed. net/

NAPOLEONI, Claudio (1962). Diccionario de Economía Política, Ediciones Castilla, Madrid, 1604 p.

NOLAN, Matt. "Lionel Robbins-definition of economics" en http/www.tvhe.co.nz/2008/10/26/quote5-lionel-robbins-definition-of-economics/ consulta 7 octubre 2013.

PIGOU, Arthur Cecil (1877-1959). www.eco-finanzas.com/economia/economistas/Arthur-Pigou-Pensamiento. Economía Neoclásica, consulta 15 septiembre 2013.

ROBBINS, Lionel (1944). Ensayo sobre la naturaleza y significación de la ciencia económica, Fondo de Cultura Económica, México.

ROBBINS, Lionel (1971). Autobiography of an Economist", Macmillan, Lenders Fondo de Cultura Económica, México.

RODRÍGUEZ, José (2013). La nueva fase de desarrollo económico y social del capitalismo mundial, Tesis doctoral accesible a texto completo en: http://www.eumed.net/tesis/jjrv/capítulolll consulta 10 octubre 2013.

SAMUELSON, Paul y NORDHAUS, William (2010). Economía con aplicaciones a Latinoamérica,Decimonovena edición, McGraw-Hill//rwin, impreso en México por Programas Educativos S.A. de C.V.

SENIOR, Nassau W. (2013). Causas y soluciones de los disturbios presentes. En:Textos Selectos de Grandes Economistas accesible libremente en www.eumed.net/textos/ consulta 11 octubre 2013.

UNIVERSIDAD DE ZARAGOZA (2011). Algunas definiciones de Economía en www.uinizar.es/departamento/ estructura_economica/.../Definición, consulta 12 agosto 2011.

UNIVERSITAT DE LES ILLES BALEARS (2013). Pensamiento económico de Cecil Pigou en http://www.ecofinanzas.com/ecoomia/economistas, consulta 31 agosto 2013.

VILLAVICENCIO, Fernando (2012). Ecuador made in China. Artes gráficas Silva, Quito, Ecuador, Segunda edición.

www.bbc.co.uk/mundoeconomía/ consulta 10 septiembre 2013.

www.kikipedia.org/wiki/Arthur_Pigou consulta 22 septiembre 2013. 